to produce genetically detoxified material. The paper by Sato et al. on mutant strains expressing pertussis toxin crossreacting materials is particularly interesting; it describes a mutant, 79G, which expressed a non-toxic material with good protective activity. The next section on "Pertussis antigen and antibody" also deals with pertussis toxin. The paper by Askelöf et al. on the immunogenic activity of a synthetic peptide corresponding to sections of the S1 subunit of pertussis toxin complements previous reports by these authors which suggest a potential role for peptide-based vaccines. It is notable, however, that the protective activity in some assays was dependent upon the mouse strain used.

No papers were presented under the heading of "Biological Standardisation for acellular pertussis vaccines" but discussion on this aspect is summarised briefly. Interestingly, the intracerebral challenge assay still seemed to be favoured as a measure of potency. On the composition of future vaccines, emphasis was placed on the use of pertussis toxoid alone rather than multi-component vaccines. This view is likely to be questioned by many involved in vaccine development. Some of these issues recur in the section on "Pertussis assay and detection systems". A useful paper by Sato et al. describes an improved ELISA method for measuring pertussis antibody. Aoyama et al. reported over $90 \%$ isolation rates of $B$. pertussis from nasopharyngeal swabs stored in Amies transport medium and inoculated on modified Bordet Gengou agar, charcoal agar or cyclodextrin solid media. This is a substantial improvement over the general experience of isolation from cases. It is not stated if the cases were selected to include only those at the earliest stage of the disease.

Subsequent sections deal with the production and properties of acellular pertussis vaccines, the human response in clinical trials and the epidemiology of the disease in Japan, Germany and Hungary. Papers of particular significance include that by Novotny et al., which provides experimental evidence in support of the protective role of the $69-\mathrm{kDa}$ protein (pertactin), and that by Klein on a phase II evaluation of various formulations of acellular vaccines containing from one to four components to be conducted in the U.S.A. This trial has now been completed but the results were not available at the time of the meeting. Other interesting observations include a study by Yabiku $e t$ al. which failed to find any evidence of a connection between vaccination with acellular pertussis vaccines and an increased susceptibility to bacterial meningitis, and an investigation of an outbreak of mixed infection with $B$. pertussis and $B$. parapertussis. The latter was only isolated from children vaccinated with pertussis vaccine or given antibiotics. This raises the possibility of an increased incidence of $B$. parapertussis infection following the suppression of $B$. pertussis in recipients of acellular vaccines.

Overall, this volume contains information which will interest the specialist reader. Its price will ensure that it is unlikely to be purchased by individuals, particularly as its contents will date very rapidly.

M. J. CORBEL

\section{Quality Control. Principles and Practice in the Microbiology Laboratory}

Edited by J. J. S. SNell, I. D. Farrell and C. RoberTS. 1991. ISBN 0901144312 . Public Health Laboratory Service. Pp. 172. A5 Paperback $£ 12.50$.

As stated in this paperback's foreword, quality control and assessment are essential aspects of laboratory medicine. The consequences of an incorrect laboratory report in microbiology, as in any other pathology discipline, are potentially serious for the patient. The need for quality control and assessment is underlined by the very nature of the science of microbiology, concerned as it is with living organisms that often display a great capacity for genetic variation. Although this book primarily covers quality control and assessment of clinical microbiology techniques, water and food microbiology-including an industrial perspective-are also covered.

The book is divided into 15 chapters each covering either general or specific areas of a microbiology laboratory. The chapters are well laid out for easy reference, in particular, the culture media section contains a very useful table giving details of a wide range of organisms that may be used to control commonly used culture media. In the chapter on the quality control of antibiotic susceptibility testing there is no mention of automated techniques and how they should be controlled. Under breakpoint dilution techniques, discussion is restricted to agar dilution methods and there is no mention of control procedures for broth dilution assays. The parasitology chapter lists the routine methods used in parasitology, and disappointingly provides little information regarding quality control. The chapter on quality control in mycology might have been better included elsewhere, as it consists of only four pages, one of which is an appendix!

The book has been written primarily with United Kingdom laboratories in mind, and might have benefited by a section on the international aspects of quality control and assessment. Nevertheless, it is comprehensive and contains a wealth of information that will be valuable to staff working in clinical microbiology laboratories. At its economical price, it should find a place as a standard reference book in every microbiology laboratory.

P. F. WHEAT

\section{Current Topics in Clinical Virology}

Edited by P. Morgan-CAPNER. 1991. ISBN 0901144304. Cambridge University Press. Pp. 304. $£ 24.95$

This is the second of a series of reviews in clinical virology published by the Public Health Laboratory Service. The topics include discussions of clinical problems, such as varicella-zoster in pregnancy, treatment of CMV infections and diagnosis of EBV-associated disease; public health problems, such as human viruses and water, and mumps vaccination; and sections on individual viruses, such as HTLV1 and Hantavirus. In addition, there are three articles on non-viral pathogens that normally come within the scope of the virology laboratory: Q-fever, chlamydia and mycoplasma. Some of the articles have appeared previously in part or whole in the PHLS Microbiology Digest, but many are newly produced.

The articles are well written and comprehensive updates. The references are generally up to date with a sprinkling of citations from 1990 and even 1991 (although these tend to be of the authors' own works). The book is produced as a paperback with good quality paper and readable print. It has a few black and white illustrations, some of which have not printed too well, but has a good collection of tables, graphs and line drawings.

The topics encompassed provide interesting reading for virologists and non-virologists alike. That the hepatitis virus and HIV are not included is acknowledged by the editor who hopes they will be included as subsequent volumes. In general, I feel it is an interesting and useful book which I would commend especially to those working for MRCPath examination.

C. A. HART 